\title{
Complex Acoustic Gravity Wave Behaviors to a Mathematical Model Arising in Nonlinear Mathematical Physics
}

\author{
Tolga Akturk ${ }^{1}$, Tukur Abdulkadir Sulaiman ${ }^{2,3}$, Haci Mehmet Baskonus $^{4}$ and Hasan \\ Bulut $^{2,5}$ \\ ${ }^{1}$ Department of Mathematics, Ordu University, Ordu, Turkey \\ ${ }^{2}$ Department of Mathematics, Firat University, Elazig, Turkey \\ ${ }^{3}$ Department of Mathematics, Federal University Dutse, Jigawa, Nigeria \\ ${ }^{4}$ Department of Computer of Engineering, Munzur University, Tunceli, Nigeria \\ ${ }^{5}$ Department of Mathematics Education, Final University, Girne, Cyprus
}

\begin{abstract}
In this article, we utilize the powerful sine-Gordon expansion method (SGEM) in constructing some new solutions to the $(2+1)$-dimensional Boiti-LeonPempinelli equation by using the Mathematica software. We successfully obtain some new travelling solutions bearing some new structures such as trigonometric function, exponential function and hyperbolic function structures. We claim that some of our results are complex in structure. All the solutions obtained verified the the $(2+1)$ dimensional Boiti-Leon-Pempinelli equation. To illustrate our results, present the numerical simulation of all the obtained solutions in this study by selecting appropriate values of the parameters. Furthermore, we give the physical interpretation of all the graphics. We also give the physical meaning to some of the obtained results in this study.
\end{abstract}

\section{Introduction}

Many aspects in nonlinear science such as mathematical physics, biological science, physics and chemistry and many more nonlinear phenomena can be explicited in the style of nonlinear partial differential equations (NPDEs). Of late years, different analytical techniques have been formulated and employed to search for some new solitary wave solutions like this models for instance the modified expansion function method [1], the generalized Kudryashov method [2], the extended homoclinic test function method [3], the simple equation method [4], the extended hyperbolic function method [5], the $\tan \left(\frac{F(\xi)}{2}\right)$-expansion method [6], the generalized tanh-function type method [7], the modified tanh-coth method [8], the generalized Bernoulli sub-equation function method [9] and many more powerful analytical methods [10-21].

\footnotetext{
* Corresponding author: tolgaakturkk@gmail.com
} 
However, in this study, we used the powerful SGEM [22, 23] in studying the solutions behaviors of the $(2+1)$-dimensional BoitiLeon-Pempinelli (BLP) equation given by [24]

$$
\begin{gathered}
u_{t y}=\left(u^{2}-u_{x}\right)_{x y}+2 v_{x x x} \\
v_{t}=v_{x x}+2 u v_{x}
\end{gathered}
$$

The (2+1)-dimensional BLP equation which arises in mathematical physics, describe the interaction of the two waves with different distributional relationships. [25].

\section{Application}

In this section, we employ the SGEM $[10,11]$ to Eq. (1.1). Performing the travelling wave transformation,

$u=u(x, y, t)=U(\xi), v=u(x, y, t)=V(\xi), \xi=\mu(\mathrm{x}+\mathrm{y}-\mathrm{ct})$ on Eq. $(1.1)$ :

$$
\mu^{2} U^{\prime \prime}-2 U^{3}-3 U^{2}-c^{2} U=0
$$

By SGEM, Eq. (3.1) is assumed to have the following solutions [10, 11]:

$$
\begin{aligned}
& \mathrm{U}(\xi)=\sum_{i=1}^{m} \tanh ^{i-1}(\xi)\left[\mathrm{B}_{i} \operatorname{sech}(\xi)+\mathrm{A}_{i} \tanh (\xi)\right]+A_{0}, \\
& \mathrm{U}(w)=\sum_{i=1}^{m} \cos ^{i-1}(w)\left[\mathrm{B}_{i} \sin (w)+\mathrm{A}_{i} \cos (w)\right]+A_{0} .
\end{aligned}
$$

we get $m=1$ by homogenous balance technique. Using Eqs. (2.2) and (2.3) with $m=1$, we obtain the following:

$$
\mathrm{U}(\xi)=B_{1} \operatorname{sech}(\xi)+\mathrm{A}_{1} \tanh (\xi)+A_{0}
$$

and

$$
\mathrm{U}(w)=B_{1} \sin (w)+\mathrm{A}_{1} \cos (w)+A_{0},
$$

respectively.

Putting Eq. (2.5) and its second derivative into Eq. (2.1), produces an equation in trigonometric functions. Equating all summation of the coefficients of the trigonometric functions of the same power to zero, we get the a set of algebraic equations. Reducing the set of algebraic equations gives the values of the coefficients inclusive. Substituting the values of the coefficients into Eq. (2.4), gives the solutions to Eq. (1.1).

\section{Case-1 :}

which gives :

$$
A_{0}=-\frac{1}{2}, A_{1}=\frac{1}{2}, B_{1}=0, \mu=\frac{1}{2}, c=1,
$$




$$
\begin{gathered}
u_{1}(x, y, t)=\frac{1}{2}\left(-1+\tanh \left[\frac{1}{2}(x+y-t)\right]\right), \\
v_{1}(x, y, t)=\frac{1}{2} \tanh \left[\frac{1}{2}(x+y-t)\right] .
\end{gathered}
$$
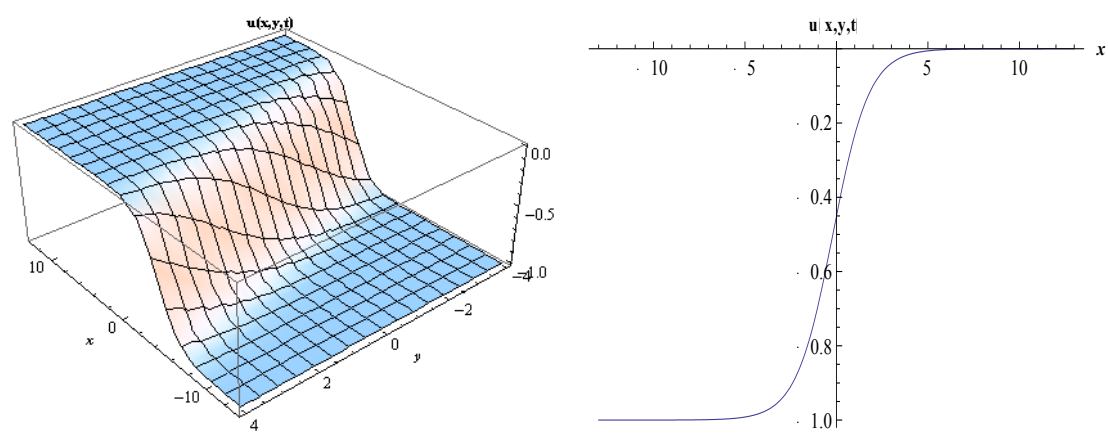

Fig.1. : The kink-type solution shape of Eq. (2.6) under the values $t=0.5,-13<\mathrm{x}<13,-2<\mathrm{y}<2$ and $y=0.7$ for the $2 \mathrm{D}$ graphic.
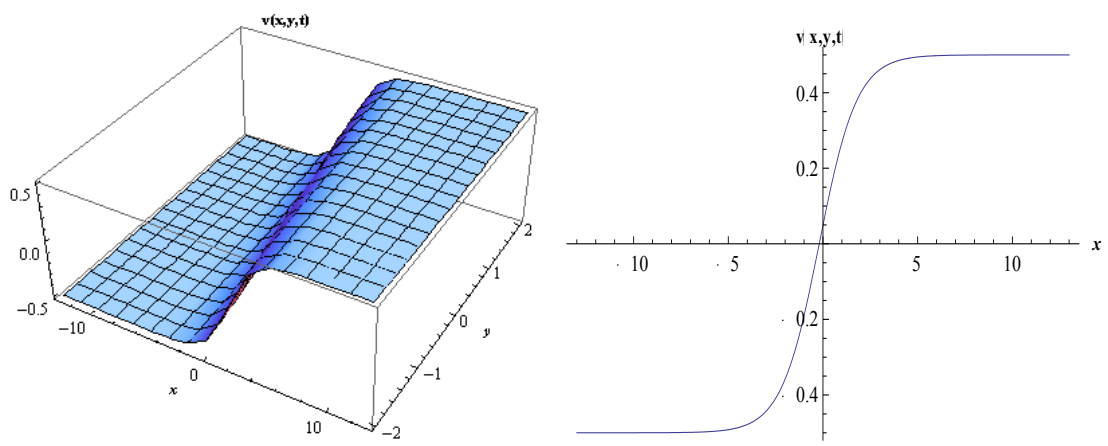

Fig. 2. : The kink-type solution shape of Eq. (2.7) under the values $t=0.5,-13<\mathrm{x}<13,-2<\mathrm{y}<2$ and $y=0.7$ for the $2 \mathrm{D}$ graphic.

\section{Case-2 :}

$$
\begin{aligned}
& A_{0}=-\frac{1}{2}, A_{1}=\frac{1}{2}, B_{1}=-\frac{i}{2}, \mu=1, c=1, \\
& u_{2}(x, y, t)=v_{2}(x, y, t)=\frac{1}{-1+i e^{x+y-t}} .
\end{aligned}
$$



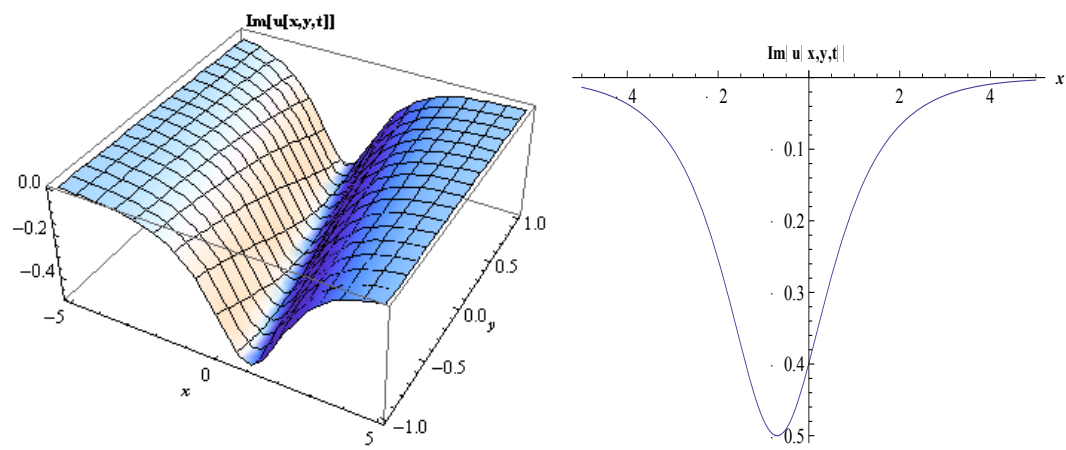

Fig. 3. : The singular solution shape of imaginary Eq. (2.8) under the values $t=0.5,-13<\mathrm{x}<13$, $-1<\mathrm{y}<1$ and $y=0.7$ for the $2 \mathrm{D}$ graphic.
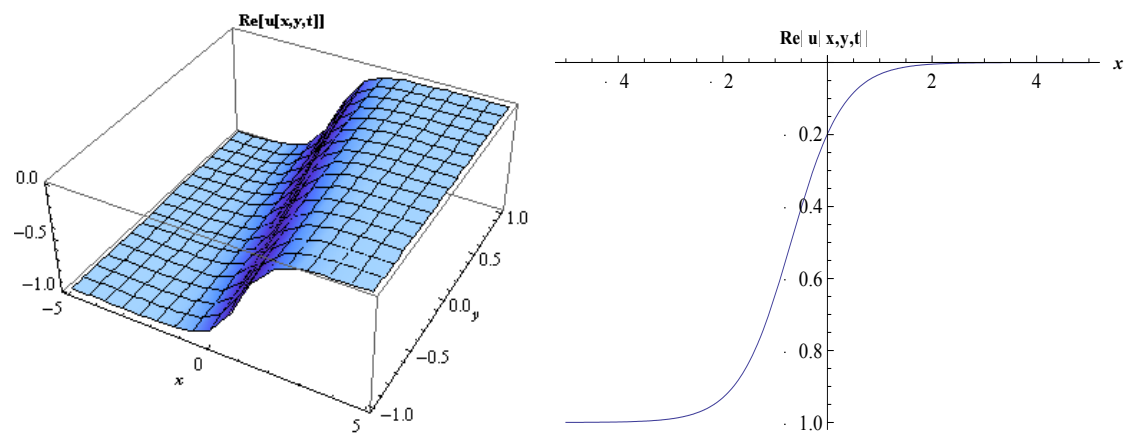

Fig. 4. : The kink-type shape of real part Eq. (2.8) under the values $t=0.5,-13<\mathrm{x}<13,-1<\mathrm{y}<1$ and $y=0.7$ for the $2 \mathrm{D}$ graphic.

\section{Conclusion}

This study is assigned in constructing some new solutions to the $(2+1)$-dimensional Boiti-LeonPempinelli equation that is used to describe the interactions of two waves with different dispersion relations in mathematical physics. We successfully found new solutions with some novel structures such as complex hyperbolic function, trigonometric function and exponential function structures. We give the physical meaning of some obtained solutions in this study. We also present the $3 \mathrm{D}$ and $2 \mathrm{D}$ surfaces of all the obtained solutions together with their physical interpretations. We compare our results with some substantial results in the literature and we sighted that our results is only just constructed results with some good structures that have important physical meaning. With these results, we can say that SGEM is indeed powerful and efficient mathematical tool that can provide you with the varieties of solution structures when employed to various nonlinear evolution equations. 


\section{References}

1. F. Ozpinar, H.M. Baskonus. H. Bulut, Entropy, 17(12), 8267-8277 (2015)

2. E.M.E. Zayed, G.M. Moatimid, A.G. Al-Nowehy, Scientific Journal of Mathematics Research, 5(2), 19-39 (2015)

3. C. Wang, Nonlinear Dynamics, 85(2), 1119-1126 (2016)

4. T.A. Nofal, Journal of the Egyptian Mathematical Society, 24, 204-209 (2016)

5. Y. Shang, Chaos, Solitons and Fractals, 36(3), 762-771 (2008)

6. J. Manafian, Optik, 127, 4222-4245 (2016)

7. W. Zhang, Applied Mathematics, 4, 11-16 (2013)

8. L. Wazzan, Communications in Nonlinear Science and Numerical Simulation, 14(6), 26422652 (2009)

9. B. Zheng, WSEAS Transactions on Mathematics, 7(11), 618-626 (2012)

10. H. Bulut, H.A. Isik, T.A. Sulaiman, ITM Web of conferences, 13, 01019 (2017)

11. S. Duran, M. Askin, T.A. Sulaiman, IJOCTA, 7(3), 240-247 (2017)

12. H.M. Baskonus, H. Bulut, A. Atangana, Smart Materials and Structures, 25(3), 035022 (2016)

13. H. Bulut, T.A. Sulaiman, H.M. Baskonus, Eur. Phys. J. Plus, 132, 459 (2017)

14. H.M. Baskonus, H. Bulut, T.A. Sulaiman, Eur. Phys. J. Plus, 132, 482 (2017)

15. H. Bulut, T.A. Sulaiman, B. Demirdag, Nonlinear Dyn, 91, 1985-1991 (2018)

16. H.M. Baskonus, T.A. Sulaiman, H. Bulut, Opt Quant Electron, 50, 14 (2018)

17. H. Bulut, T.A. Sulaiman, H.M. Baskonus, T. Akturk, Opt Quant Electron, 50, 19 (2018)

18. T.A. Sulaiman, T. Akturk, H. Bulut, H.M. Baskonus, Journal of Electromagnetic Waves and Applications, 32(9) 1093-1105 (2017)

19. A. Yokus, T.A. Sulaiman, H. Bulut, Opt Quant Electron, 5031 (2018)

20. S.Ş. Şener, Y Saraç, M. Subaşı, Applied Mathematical Modelling, 37, 2623-2629 (2013) 
21. M. Subaşı, S.Ş. Şener, Boundary Value Problems, 1-16 (2015)

22. C. Yan, Physics Letters A, 22(4), 77-84 (1996)

23. H. Bulut, T.A. Sulaiman, F. Erdogan, H.M. Baskonus, Eur. Phys. J. Plus, 132, 350 (2017)

24. H.M. Baskonus, H. Bulut, Waves in Random and Complex Media, 26(2), 201-208 (2016)

25. M. Kumar, R. Kumar, A. Kumar, Computers and Mathematics with Applications, 70, $212-$ $221(2015)$ 